\title{
Bedsores Incidence in ICU Patients: A Tertiary Care Experience
}

\author{
Qurat-ul-Ain Khan* \\ Critical Care Department, Ziauddin University Hospital, Karachi, Pakistan.
}

\begin{abstract}
Introduction: The aim of this study was to see the incidence of bedsores in our hospital and determine relating factors.
Material and Methods: This cross sectional study was performed on 71 consecutive ICU admissions at Ziauddin hospital ICU from March to May 2015. Data was analyzed on SPSS version 16 by independent sample t-test and chi square test. In 71 patients, 12 developed bedsores, of which seven were males and five were females. Available data in Performa included: demographics, functional class, comorbidities, category(surgical, medical or trauma patient), primary cause of ICU admission, total day of ICU stay, total period of mechanical ventilation, requirement of dialysis, albumen level and if bedsore developed than its details and grade.
\end{abstract}

Result: In this study, 71 patients were admitted in ICU; 40 patients, which is $56.33 \%$, were male and 31 patients, which is $43.66 \%$, were female. Twelve patients that are $16.90 \%$ were affected with bedsores of which seven were males and five were females and this was found to be statistically significant.

The mean age of patients with bedsores was 66.75 years \pm 18.29 as compared to 52.42 years \pm 18.20 in group with no bedsore development.

Total mechanical ventilation required in the group that developed bedsore was 4 days \pm 1.67 to group with no bedsore development, which was 2.36 days \pm 1.21 .

The patients who developed bedsore had serum albumen levels $2.54 \pm 0.26$ and group that did not develop bedsore had albumen levels $3.14 \pm$ 0.77 and the result was found to be statistically significant.

Duration of Study: From June 2017 to June 2018.

Conclusion: Development of bedsore was found to be statistically significant to the poor functional class, high mean age, total hospital stay, mean mechanical ventilation and low level of albumin.

Keywords: Bedsores, Pressure ulcers, Hospital, Admitted patients, Intensive care unit, Incidence.

\section{INTRODUCTION}

Pressure ulcers are a result of added pressure on skin's capillary - arterial system. Such ulcers heal slowly and if not managed may lead to local and systemic infection add to morbidity and mortality. Its development is a major concern in bedridden, poor functional class and ICU patients. Many may be associated with poor medical condition or additional surgical problem [1] that makes recovery and mobility slow. Pressure is ischemia or necrosis of skin as result from compression from external surface and osteoid process for a prolonged period [2]. The relating factors are old age, presence of malnutrition, dehydration, prolonged illness, overpressure and friction on skin [3]. Bedsore frequently involves immobile and unconscious patients who have no feeling of pain [1]. The commonly involved sites are sacrum-pelvis, occipital area, and heel as they are under more compression [1]. ICU patients are further susceptible due to poor functional class. Immobility, mechanical ventilation and due to effect of sedative $[4,5]$ skin care in ICU patients should

*Address correspondence to this author at the Critical Care Department, Ziauddin University Hospital, Karachi, Pakistan.

E-mail: quratkhan@hotmail.com be paid attention to particularly to susceptible anatomic areas of bedsore development and if they occur, should be appropriately managed. High incidence of bedsore development is considered a poor score for the hospital [6] as it can increase morbidity and mortality [7]. Several studies have shown increased bedsore variables in ICU from $3.8 \%$ to $33.3 \%[5,8,9]$. Frequency has also been known to increase in trauma patient [10]. Despite increase in healthcare services, the frequency of bedsore has increased and thus is a major concern for critical care teams [2, 3, 11]. Patients in need of critical care predisposed them to the development of bedsores and for their management is difficult, accurate programming should be developed. To the best of our knowledge, there is no such study in our area, hence decided to conduct one.

\section{MATERIAL AND METHODS}

TThe study design is a prospective cohort with a sampling technique being purposive sampling done in our hospital's ICU patients after taking clearance from our hospitals Ethical Review Committee, after taking informed consent from next of kin and attendants. This is a study carried out from a period of March to May 2015 on 71 ICU admissions. An exclusion 
criterion was prior bedsore and discharge from ICU in 24 hours. The data were assigned to all surgical and medical patients in ICU who did not fall in exclusion criteria and the development of a bedsore in any was noted and assessed. Available data in Performa included: demographics, functional class, comorbidities, category(surgical, medical or trauma patient), primary cause of ICU admission, total day of ICU stay, total period of mechanical ventilation, requirement of dialysis, albumen level and if bedsore developed than its details and grade. European Ulcer Advisory Panel grading system for bedsore was used which is as follows: Stage 1: piece of normal visible skin distinguishable from surrounding by temperature, tissue stability and sensitivity. Stage 2: partial loss of epidermal or dermal layer or both. Stage 3: Total loss of cutaneous layer including subcutaneous lesion and necrosis and progression into cutaneous levels. Stage 4: complete loss of cutaneous layer, along with damage to muscles bones, and protective structures [12]. Data collected on Performa was gathered and analyzed on SPSS version 16. Mean and standard deviation were reported for age, total hospital stay, mechanical ventilation, albumen levels, bedsores and no bedsores and degree of bedsores, Independent sample t-test was used for statistical mean difference in both group. Frequency and percentage were also reported for gender, BMI, number of commodities and infections. Peterson chi-square test was used to see the association with bedsores and the degree of bedsore. Results with a $\mathrm{p}$ value less than 0.05 were considered significant.

\section{RESULTS}

In this study, 71 patients were admitted in ICU; 40 patients, which is $56.33 \%$, were male and 31 patients, which is $43.66 \%$, were female. Twelve patients that are $16.90 \%$ were affected with bedsores of which seven were males and five were females and this was found to be statistically significant. The mean age of patients with bedsores was 66.75 years \pm 18.29 as compared to 52.42 years \pm 18.20 in group with no bedsore development and it was found to be statistically significant. Most of the patients admitted were under medical ICU team 57, while surgical patients were 14. Fifty five patients from both groups were admitted from emergency department as compared to admissions from wards, which were 16. Most of the patients, 59 admitted had BMI 18.5 to 25 but it was not statistically significant. All patients who developed bedsores had grade 2 bedsores and were found at sacral and iliac region.

Chi-square results of relationship between presence or absence of bedsore to BMI was bedsore occurring mostly in 18.5-25 and was not significant. Most of the patients who developed bedsore had nonnative functional class $54.5 \%$, while those who did not have bedsore had active functional class $80.7 \%$ and it was found to be statistically significant. Most of the patients who developed bedsores had two $41.70 \%$ or more than two comorbidities $41.70 \%$ as compared to those who did not develop bedsore with most of patients in this group having only one comorbidity, $40.70 \%$. Most of the patients in both groups had single infection. Higher percentage of patients who developed bedsores required dialysis. Thirty percent of the patients who developed bedsores as compared to $8.9 \%$ from non-bedsore group died in ICU.

Independent sample test results were seen for mean age, total hospital stay, total mechanical ventilation required and level of albumen to patients developing bedsore to those not developing bedsores. The mean age of patients developing bedsores was 66.75 years \pm 18.29 compared to those not developing bedsores, $52.42 \pm 18.20$ and it was found to be statistically significant. The patients who developed bedsores had a hospital stay of 10.58 days \pm 1.96 as compared to those not developing bedsore 8.62 days \pm 5.42 , it was not statistically significant. Total mechanical ventilation required in the group that developed bedsore was 4 days \pm 1.67 to group with no bedsore development, which was 2.36 days \pm 1.21 and it was statistically significant. The patients who developed bedsore had serum albumen levels $2.54 \pm 0.26$ and group that did not develop bedsore had albumen levels $3.14 \pm$ 0.77 and the result was found to be statistically significant. All patients with bedsores were managed conservatively and none required surgical intervention with debridement under GA.

\section{DISCUSSION}

The purpose of our study, which is the first of its kind in the country to the best of our knowledge, was to look at the incidence of bedsore in patients admitted in our hospital and its association with different factors. The incidence of bedsore occurrence in our admitted patients was $16.90 \%$. This incidence was quite comparable to $14 \%$ in hospitalized patients according Braden criteria [13], 19.7\% from a study by Hagiswa [14] and $19.1 \%$ according to Mogharehi in hospital in Iran [15]. The incidence of bedsore was slightly less in study by Nayak D [16] which fell in range of 3-11\%.

The region of bedsore of all of our patients was at sacrum and iliac area. This finding was similar to what was mentioned by studies from Lingren [17] and Tubaishat [18] but along sacrum they also mentioned heel to be equally common. Soozani and Hassani mentioned iliac tuberosity and sacrum to be the most frequent site. Sacrum was also mentioned to be the most common site [19]. The reason of development of bedsores at sacrum region in our study and other studies that looked for incidence of bedsore in ICU patients was due to the fact the sacral region experiences highest pressure as the patient is lying straight with upward held head and torso.

The degree of bedsore in our entire patient was grade 2 and it is in contrast to another study done on trauma ICU patients [19] where all grade of bedsore was found. 
It is obvious that the most important factor in development of bedsore was constant pressure and immobility. Other factors with a possible relationship have been looked into. In the current study, there was no significant relationship with BMI, gender, increased number of comorbidities, number of infections and requirement of dialysis. This was also consistent with [19], however age mentioned as a nonsignificant association in this study [19] and by Reyhani-e-Kermani [20] was not similar our results in which we found significant association with age. Study by Gallagher et al. [21] increased duration of hospital stay was significantly associated with bedsore development and this was a consistent feature of study done on trauma ICU patients [12]. Low level of serum albumen was also significantly associated with bedsore development in our study.

The most important factor of development of a bedsore is persistent pressure on cutaneous layers and hampering blood circulation leading to ulceration. The management therefore mainly involves regular monitoring of susceptible areas and sequential displacement, particularly for unconscious ICU patients to prevent the development of bedsore. Adequate preparation should be done in patients likely to develop bedsores.

Pressure sore can be prevented by identifying high-risk group. One should focus on correcting nutritional status, frequent position change and to use a bedsore mattress in high - risk groups. One can use of emollients for dry skin or skin barrier products if it is excessively moist.

All of our patients who developed bedsores required were managed conservatively by wound dressing and care, changing position and by providing bedsore mattress, infection control, pain management and correcting the main disease. Only a few patients required minimal debridement at bedside. We further added protein in the diet. All these factors prevented the progress of the bedsore stage with all those who had it.

\section{CONCLUSION}

Bedsore development is a common occurrence in ICU patient. Development of bedsore was found to be statistically significant to the poor functional class, high mean age, total hospital stay, mean mechanical ventilation and low level of albumin. Presence of bedsore should be actively monitored and appropriately managed.

\section{CONFLICT OF INTEREST}

Declared none.

\section{ACKNOWLEDGEMENTS}

Declared none.

\section{REFERENCES}

[1] Chico-Fernández M, Terceros-Almanza L, Mudarra-Reche C. Innovation and new trends in critical trauma disease. Med Intensiva (English Edition) 2015; 39(3): 179-88.

DOI: $10.1016 /$ j.medine.2015.03.002

[2] Potter PA, Perry AG, Hall A, Stockert PA. Fundamentals of Nursing. USA: Elsevier Mosby 2009.

[3] Terekeci H, Kucukardali Y, Top C, Onem Y, Celik S, Öktenli Ç. Risk assessment study of the pressure ulcers in intensive care unit patients. Eur J Int Med 2009; 20(4): 394-7.

DOI: $10.1016 /$ j.ejim.2008.11.001

[4] Keller PB, Wille J, van Ramshorst B, van der Werken C. Pressure ulcers in intensive care patients: A review of risks and prevention. Intens Care Med 2002; 28(10): 1379-88. DOI: $10.1007 / \mathrm{s} 00134-002-1487-\mathrm{z}$

[5] Elliott R, McKinley S, Fox V. Quality improvement program to reduce the prevalence of pressure ulcers in an intensive care unit. Am J Crit Care 2008; 17(4): 328-34.

[6] Sanada H, Sugama J, Thigpen B, Kitagawa A, Kinosita S, Murayama S. A new instrument for predicting pressure ulcer risk in an intensive care unit. J Tissue Viability 2006; 16(3): 216.

[7] Yoo Y, Mun S. The advantages of early trauma team activation in the management of major trauma patients who underwent exploratory laparoto-my. Ann Surg Treat Res 2014; 87(6): 319-24. DOI: 10.4174/astr.2014.87.6.319

[8] Cho I, Noh M. Braden scale: Evaluation of clinical usefulness in an intensive care unit. J Adv Nurs 2010; 66(2): 293-302. DOI: $10.1111 /$ j.1365-2648.2009.05153.x

[9] Shahin ES, Dassen T, Halfens RJ. Pressure ulcer prevention in intensive care patients: Guidelines and practice. J Eval Clin Pract 2009; 15(2): 370-4.

DOI: $10.1111 /$ j.1365-2753.2008.01018.x

[10] Shahin ES, Dassen T, Halfens RJ. Pressure ulcer prevalence and incidence in intensive care patients: A literature review. Nurs Crit Care 2008; 13(2): 71-9. DOI: $10.1111 /$ j.1478-5153.2007.00249.x

[11] Fernandes LM, Caliri MHL, Haas VJ. The effect of educative interventions on the pressure ulcer prevention knowledge of nursing professionals. Acta Paul Enferm 2008; 21(2): 305-11. DOI: $10.1590 / \mathrm{S} 0103-21002008000200012$

[12] Toner L. European pressure ulcer advisory panel. $\mathrm{Br} \mathrm{J}$ Commun Nurs 2007; 12(Sup4): S3-S.

[13] Jamand T, Akaberian S, Khoramroodi R. Assessment of risk factors associated with bedsores in patients admitted to Fatemeh Zahra \& Salman Farsi Hospitals in Boushehr by using the braden indices (2007-2008). ISMJ 2012; 15(3): 233-40. 
[14] Hagisawa S, Ferguson-Pell M. Evidence supporting the use of two-hourly turning for pressure ulcer prevention. J Tissue Viability 2008; 17(3): 76-81. DOI: 10.1016/j.jtv.2007.10.001

[15] Mogarehi M, Sanaiey Z. Pressure sore incidence and risk factors in patients admitted in medical surgical and orthopedic wards in affiliated hospitals of Shiraz University of medical sciences. Iran J Nurs 2003; 16(34): 8-13.

[16] Nayak D, Srinivasan K, Jagdish S, et al. Bedsores:"Top to bottom" and "bottom to top". Indian J Surg 2008; 70(4): 161-8. DOI: $10.1007 / \mathrm{s} 12262-008-0046-4$

[17] Lindgren M, Unosson M, Krantz A, Ek A. Pressure ulcer risk factors in patients undergoing surgery. J Adv Nurs 2005; 50(6): 605-12. DOI: 10.1111/j.1365-2648.2005.03441.x
[18] Mafraq J. The prevalence of pressure ulceration among Jordanian hospitalised patients. J Wound Care 2013; 22(6): 305. DOI: 10.12968/jowc.2013.22.6.305

[19] Farshid RB, Hamed S, Saadat T. Bedsore incidence in trauma patients admitted to the ICU. J Biol Today's World 2014; 3(12): 261-5.

[20] Soozani A, Hasani M. The effect of structured educational model on knowledge of caregivers and the process of pressure ulcer healing in veterans with spinal cord injury in Shahroud, Iran. Horizon Med Sci 2007; 12(4): 55-61.

[21] Reyhani H, Haghiri A. Determination of bed sore risk factors in cranio-spainal trauma patient in intensive care unit. Arak J Med Sci 2006; 10(2): 79-84.

(C) 2019 National Journal of Health Sciences.

This is an open-access article. 